\title{
Maternal diet quality and nutrient intake in the gestational period: results from the delta healthy sprouts comparative impact trial
}

Lisa M. Tussing-Humphreys ${ }^{1 *}$, Jessica L. Thomson², Melissa H. Goodman² and Sarah Olender ${ }^{1}$

\begin{abstract}
Background: A woman's diet while pregnant can play an important role in her reproductive health as well as the health of her unborn child. Diet quality and nutrient intake amongst pregnant women residing in the rural Lower Mississippi Delta (LMD) region of the United States is inadequate. The Delta Healthy Sprouts Project was designed to test the comparative impact of two home visiting programs on weight status, dietary intake, and health behaviors of women and their infants residing in the LMD region. This paper reports results pertaining to maternal diet quality and nutrient intake in the gestational period.
\end{abstract}

Methods: The experimental arm (PATE) received monthly home visits beginning in the second trimester using the Parents as Teachers curriculum enhanced with a nutrition and lifestyle behavior curriculum. The control arm (PAT) received monthly home visits using the Parents as Teachers curriculum only. Maternal diet was assessed via 24-h dietary recall at gestational months (GM) 4 (baseline), 6, and 8. Diet quality was computed using the Healthy Eating Index-2010 (HEl-2010).

Results: Gestational period retention rates for PAT and PATE arms were 77 \% (33/43) and $67 \%$ (26/39), respectively. Significant effects were not found for time, treatment, or time by treatment for the HEl-2010 total or component scores, macro- or micronutrient intake or percentage of women meeting recommended nutrient intakes.

Conclusions: Perhaps due to low participant enrollment and higher than expected rates of drop out and noncompliance, we were not able to demonstrate that the enhanced nutrition and lifestyle curriculum (PATE) intervention had a significant effect on diet quality or nutrient intake during pregnancy in this cohort of rural, Southern, primarily African American women.

Trial registration: clinicaltrials.gov, NCT01746394. Registered 5 December 2012.

Keywords: Maternal diet quality, Pregnancy, African American women

\section{Background}

A woman's diet throughout pregnancy can play an important role in her reproductive health as well as the health of her unborn child [1]. Modest increases in energy intake in the $2^{\text {nd }}$ and $3^{\text {rd }}$ trimester and greater intake of several micronutrients including iron and folate throughout the gestational period are necessary to

\footnotetext{
*Correspondence: Itussing@uic.edu

'Department of Medicine and Cancer Center, University of Illinois at Chicago, 1747 W Roosevelt Road, \#416, Chicago, IL 60618, USA

Full list of author information is available at the end of the article
}

support a healthy pregnancy. Sub-optimal maternal nutrition is linked to adverse pregnancy outcomes including excessive maternal weight gain, the development of gestational complications including gestational diabetes mellitus, increased rates of preterm birth, infant growth restriction, and maternal and infant morbidity and mortality $[2,3]$.

Historically, research examining maternal diet has focused on the adequate intake of single nutrients during the gestational period $[4,5]$. Although specific nutrients 
are indeed critical during pregnancy, nutritional research has shifted away from examining single nutrients and has begun to focus on examining the relationship between overall diet, specifically maternal diet quality, and maternal and fetal health [6]. This approach allows for the examination of total diet and the synergy of nutrients eaten together from various foodstuffs [7]. Relatively few studies have examined associations between maternal diet quality and pregnancy related outcomes [5]. However, there is some evidence that consuming a higher quality diet in the gestational period is associated with a reduction in maternal depressive symptoms [8], lower maternal fasting plasma glucose and reduced risk for gestational diabetes mellitus [9], and reduced risk for preeclampsia [10]. A recent summary of the limited but existing evidence suggests that a high-quality maternal diet consumed throughout the gestational period may reduce the risk for preterm birth, infant growth restriction, and fetal anomalies including neural tube defects [5]. Thus, interventions designed to optimize maternal diet during the gestational period have the potential to positively impact both maternal and fetal health.

One method to assess diet quality in pregnancy is to determine a woman's adherence to the Dietary Guidelines for Americans (DGAs) [11] using the Healthy Eating Index (HEI) [12]. The HEI was designed by the United States Department of Agriculture, Center for Nutrition Policy and Promotion, to monitor diet quality in the United States (US) population and is appropriate for monitoring diet quality in at-risk low income subpopulations. The HEI ranges from 0 to 100 points with scores above 80 points indicative of a higher quality diet. In non-pregnant adults, a higher HEI score has been associated with lower total and cause-specific mortality [13] and lower prevalence of metabolic syndrome [14]. The HEI has been used successfully to assess diet quality in pregnancy [15]. However, Pick and colleagues noted that HEI failed to pick up inadequate micronutrient intake in a cohort of pregnant women. This finding suggests that when measuring diet quality in pregnancy, HEI may need to be modified to include key micronutrients (i.e., iron, folate, and calcium) $[3,16]$ or used in conjunction with more detailed nutrient intake data $[17,18]$.

The Lower Mississippi Delta (LMD) region of Mississippi is characterized by low education attainment and high rates of both poverty and chronic diseases including hypertension and obesity [19] and constitutes a majority African American population. Reproductive age women residing in this region suffer from some of the highest rates of adverse pregnancy-related outcomes in the US including maternal mortality, infant mortality, preterm birth, and low infant birth weight $[20,21]$. Women of lower socioeconomic status, such as those residing in the LMD region, are at risk of being undernourished when they enter pregnancy due to poor diet and shorter intervals between pregnancies which can adversely impact a woman's nutritional state both preconception and during pregnancy [22]. We recently reported that maternal diet quality and nutrient intake in the early $2^{\text {nd }}$ trimester amongst pregnant women residing in the LMD region is strikingly inadequate [23]. Overall, these women had very poor adherence to the 2010 DGAs based on HEI, and only a limited number of women met nutrient intake recommendations for fiber, sodium, calcium or choline. This was largely attributed to their low consumption of greens and beans, fruit, whole grains, and seafood and plant proteins - foods which have relatively high micronutrient and fiber, and low saturated fat and sodium contents. Given that poor maternal nutrition can affect not only the woman but her growing fetus, the diets of these women deserve considerable attention. Clearly, there is a need for interventions in the LMD region that are designed to help women achieve optimal nutrition through improved diet quality and nutrient intake throughout pregnancy.

The Delta Healthy Sprouts Project was designed to test the comparative impact of two maternal, infant, and early childhood home visiting programs on weight status, dietary intake, and health behaviors of women and their infants residing in the rural LMD region of Mississippi [24]. Results of the primary outcome, gestational weight gain, have been previously reported (Thomson, under review). This paper describes and compares the dietary intake outcomes of Delta Healthy Sprouts participants during the gestational period.

\section{Methods}

\section{Design and recruitment}

This was a longitudinal analysis of the Delta Healthy Sprouts participants' diet behaviors measured at the baseline [enrollment; gestational month (GM) 4] visit and two subsequent gestational (GM 6 and 8) visits. A comprehensive description of the Delta Healthy Sprouts Project has been published elsewhere [24]. Briefly, 82 pregnant women were enrolled in this on-going project conducted in three LMD counties. Inclusion criteria included female gender, at least 18 years of age, less than 19 weeks pregnant with first, second or third child, singleton pregnancy, and resident of Washington, Bolivar, or Humphreys County in Mississippi. Participant enrollment occurred on a rolling basis; hence baseline data were collected between March 2013 and December 2014. The target enrollment was 75 women in each of the two arms (control and experimental) of the project. The sample size of 150 women was based on the following assumptions: $20 \%$ attrition rate, $37 \%$ of control participants with GWG within the Institute of Medicine (IOM) recommendations, and a $22 \%$ difference between treatment arms for GWG within recommendations [24]. However, recruitment was stopped by the study's 
Principal Investigator prior to reaching these numbers due to unexpected difficulties recruiting pregnant women meeting study criteria. Recruitment was extended as long as possible, but fiscal issues eventually necessitated the closing of this period. Figure 1 illustrates the CONSORT diagram.

The project was approved by the Institutional Review Board of Delta State University, Cleveland MS (\#12-024) and all participants gave written informed consent prior to any study engagement. Delta Healthy Sprouts is registered at clinicaltrials.gov (NCT01746394).

Delta Healthy Sprouts is evaluating the impact of the Parents as Teachers ${ }^{\circ}$ (PAT) curriculum compared to a nutrition and physical activity enhanced PAT curriculum (PATE) on the primary outcomes of maternal gestational weight gain and postpartum weight control and childhood obesity prevention. Secondary outcomes pertain to maternal diet quality and nutrient intake during the gestational and postpartum periods, maternal physical activity, breast feeding and child feeding practices and other child-health related outcomes. Parents as Teachers is a nationally recognized, evidence based, home visiting program that seeks to increase parental knowledge of child development, improve parenting practices, provide early detection of developmental delays, prevent child abuse, and increase school readiness [25]. Participants were randomly assigned to one of two treatment arms [PAT control $(n=43)$ or PATE experimental $(n=39)]$ and are being followed for 18 months ( 6 months gestation and 12 months postnatal). At the baseline (GM 4) visit, demographic data and anthropometric measures were collected, 24-h dietary recalls were conducted, and physical activity and other questionnaires were administered.

\section{Intervention}

The control arm of the intervention is based on the PAT curriculum that includes one-on-one home visits, developmental screenings, and a resource network for families. Home visitation is the key component of the

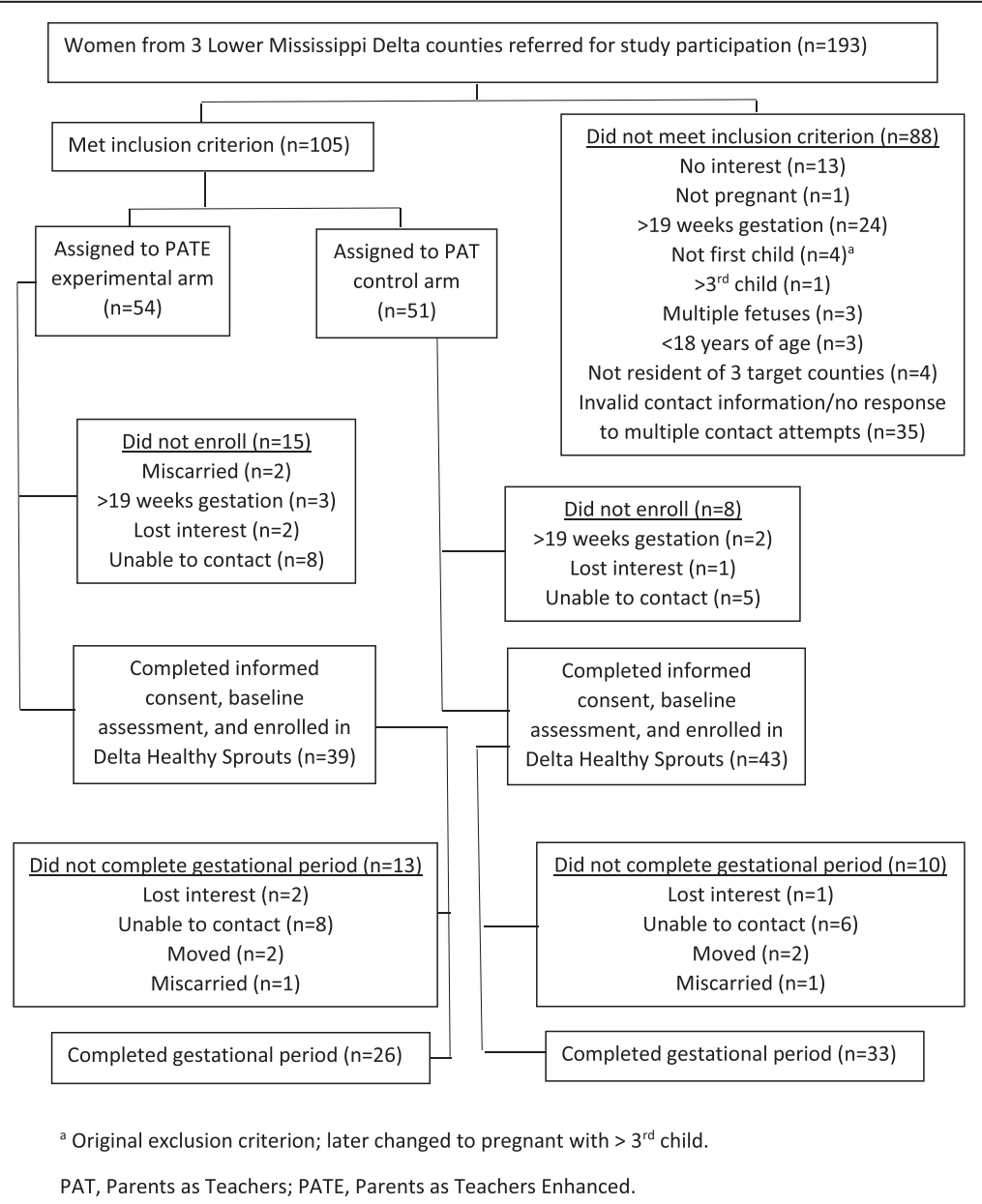

Fig. 1 CONSORT diagram of recruitment, assignment, enrollment, and completion of gestational period for Delta Healthy Sprouts Project. ${ }^{a}$ Original exclusion criterion; later changed to pregnant with $>3^{\text {rd }}$ child 
PAT model where Parent Educators provide parents with research based information and activities. Materials are tailored to the age of the child and are responsive to parental information requests.

The experimental arm of the intervention builds upon the PAT curriculum by adding culturally tailored, maternal weight management and early childhood obesity prevention components. These features are based upon foundational elements from the Diabetes Prevention Program (DPP) and the Infant Feeding Activity and Nutrition Trial (InFANT). Elements based upon the DPP principles included a flexible, culturally sensitive, individualized educational curriculum taught on a oneto-one basis [26]. Elements taken from InFANT included anticipatory guidance and parenting support principles [27]. Anticipatory guidance involves providing practical, developmentally appropriate, child health information to parents in anticipation of significant physical, emotional, and psychological milestones [28]. Parenting support emphasizes children's psychological and behavioral goals, logical and natural consequences, mutual respect, and encouragement techniques [28, 29].

For PATE, emphasis is placed on educating mothers about the ways in which they can facilitate the development of appropriate eating, physical activity, and other health behaviors in their children, including modeling these behaviors themselves. Intervention components of the PATE arm included appropriate weight gain during pregnancy and weight management after pregnancy, nutrition and physical activity in the gestational (mother) and postnatal (mother and infant) periods, breastfeeding, appropriate introduction of solid foods, and parental modeling of positive behaviors. Lessons included handson activities, instructional DVDs, and goal setting and reducing barriers for both diet and exercise. At each monthly visit in the gestational period, participants were given weight gain charts that contained reference ranges from the Institute of Medicine gestational weight gain recommendations. Participants' current as well as past weight gain from previous visits was marked on these charts.

Both arms of the intervention were delivered in the home to women beginning in their early second trimester of pregnancy by trained community based Parent Educators. Parent Educators were African American college educated women residing in the target communities. They were trained to deliver the nutrition and physical activity lessons and to collect data from participants, including dietary intake. Home visits occurred monthly and were approximately $60-90 \mathrm{~min}$ in length for the PAT lessons, and approximately 90-120 min for the PATE lessons. Additional details regarding Parent Educator training, study methodology and lesson plan outlines have been published elsewhere [24].

\section{Measures}

Anthropometric measures obtained on the participants at the baseline visit included height which was measured in duplicate using a portable stadiometer (model seca 217, seca, Birmingham, UK), and weight which was measured using a digital scale (model SR241, SR Instruments, Tonawanda, NY). Both measures were performed without shoes or heavy clothing. Pre-pregnancy weight was self-reported. Body mass index (BMI) was calculated as weight $(\mathrm{kg})$ divided by height $(\mathrm{m})$ squared where height was averaged if the two measurements differed. Weight also was measured at each of the five subsequent gestational visits. Weight gain is examined in another paper (Thomson, under review).

Dietary data were collected from the participants at the baseline, GM 6, and GM 8 visits via multiple pass 24-h dietary recall using Nutrition Data System for Research (NDSR) software. NDSR is a Windows-based dietary analysis program that allows for the calculation of nutrients per ingredient, food, meal, and day in report and analysis formats [30]. The software also includes a dietary supplement assessment module so that nutrient intake from supplemental sources can be captured and quantified [31]. Using the dietary data collected with NDSR, participants' diet quality was calculated using the Healthy Eating Index-2010 (HEI-2010) [12], which measures adherence to the 2010 DGAs. The HEI-2010 includes 12 components that are summed to create a total score ranging from 0 to 100 points. The 12 components include: total vegetables, greens and beans, total fruit, whole fruit, whole grains, dairy, total protein foods, seafood and plant protein foods, fatty acids, refined grains, sodium, and empty calories. For each component, higher scores reflect greater adherence to the 2010 DGAs. An HEI score greater than 80 is representative of a good quality diet; $51-80$ is consistent with a diet that needs improvement in regard to quality; and a score less than 51 represents a poor quality diet [32]. Additionally, intakes of specific nutrients of interest were determined using the dietary data collected. These nutrients (total and saturated fat, cholesterol, sodium, calcium, fiber, folate, iron, vitamins C and D, and choline) were chosen based on their importance to an overall healthy diet and increased nutrient needs during pregnancy [33-37].

Participants also provided information regarding demographic characteristics (e.g., age, marital status, household size, education, employment, household income, insurance, prenatal care), health history, and current health conditions. Details regarding other measures and questionnaire data that were collected using validated tools, but are not relevant to the current paper, have been published elsewhere [24]. All measures, dietary recalls, and questionnaires were collected or administered by trained research staff (Parent Educators) using 
laptop computers loaded with relevant software (i.e., NDSR and Snap Surveys).

\section{Statistical analyses}

Statistical analyses were performed using $\mathrm{SAS}^{\bullet}$ software, version 9.4 (SAS Institute Inc., Cary, NC). Descriptive statistics, including means, standard deviations, frequencies, and percentages, were used to summarize participants' demographic characteristics, anthropometric measures, nutrient intake, and diet quality.

Chi square tests of association or Fisher's exact tests (categorical measures) and two sample t tests (continuous measures) were used to assess differences between PAT and PATE participants' baseline characteristics and between gestational period study completers' and noncompleters' baseline characteristics. Study completers were defined as participants who had their GM 9 visit or those who had at least two visits in the gestational period and their post-natal 1 visit. The second definition was used because a substantial proportion of PAT and PATE participants (36 and $42 \%$ ) who had their postnatal 1 visit, missed their GM 9 visit due to the early birth of their infant.

Compliance with diet recommendations was determined based on current guidelines set by professional organizations. Participants were classified as compliant with dietary guidelines if they met minimum daily intake for fiber $(28 \mathrm{~g})$, calcium $(1300 \mathrm{mg}$ for women 18 years of age and $1000 \mathrm{mg}$ for women $\geq 19$ years of age), folate $(600 \mathrm{mcg})$, iron $(27 \mathrm{mg})$, vitamin C (80 $\mathrm{mg}$ for women 18 years of age and $85 \mathrm{mg}$ for women $\geq 19$ years of age), vitamin D $(5 \mathrm{mcg})$, and choline $(450 \mathrm{mg})$ or did not exceed the maximum daily intake for total dietary fat (35 \% of total energy), saturated fat (10\% of total energy), cholesterol (300 mg), and sodium (1500 mg for African American women).

Generalized linear mixed models, using maximum likelihood estimation, were used to test for significant treatment, time, and treatment by time (interaction) effects on diet outcomes. Maximum likelihood estimation is an approach for handling missing data in repeated measures. Treatment (PAT vs. PATE) was modeled as a fixed effect and time (GM 4, GM 6, and GM 8 visits) was modeled as a repeated measure using a first-order autoregressive covariance matrix structure. If overdispersion was present, then a multiplicative overdispersion parameter was added to the model. Least squares means with $95 \%$ confidence intervals were computed using these models. Distributions of the diet outcome variables were checked for approximate normality based on both goodness of fit tests (Cramer-von Mises and AndersonDarling) and visual inspection. If the distribution passed the goodness of fit tests or failed the tests but appeared sufficiently normal for the underlying assumptions of normality to be reasonable for practical purposes of analysis, then the diet outcome (HEI total and component scores; and total fat, sodium, folate, and vitamin D intakes) was modeled using a Gaussian distribution with an identity link function. The remaining variables were checked for approximate gamma, inverse normal, and log normal distributions, again based on goodness of fit tests and visual inspection. Saturated fat and calcium intakes were modeled using a gamma distribution with a log link function. The remaining five diet outcome variables (cholesterol, dietary fiber, iron, vitamin $C$, and choline) were modeled using quantile regression on the median to test for significant treatment, time and treatment by time effects. Treatment and time were modeled as fixed effects and $95 \%$ confidence intervals for the medians were computed using Markov chain marginal bootstrap resampling. Generalized linear mixed models also were used to test for significant treatment, time, and treatment by time effects on the proportions of participants who met recommended nutrient intakes using a binomial distribution with a logit link function. The significance level of the tests was set at 0.05 .

\section{Results}

Study retention rates during the gestational period for the PAT and PATE treatment arms were $77 \%(33 / 43)$ and $67 \%$ (26/39), respectively, and did not differ between arms $(p=$ 0.310). Compliance rates for the GM 6 and GM 8 visits were 88 and $84 \%$, respectively, for PAT participants, and 67 and $51 \%$, respectively, for PATE participants. Compliance rates for both visits were significantly lower in the PATE arm ( $p=0.018$ and 0.002 , respectively). One PAT participant did not provide dietary intake data at the GM 4 (baseline) visit. One PATE participant who missed her GM 6 visit provided dietary intake data during her GM 7 visit. Likewise, two PATE participants who missed their GM 8 visit provided dietary intake data during their GM 9 visit.

Table 1 presents comparisons between treatment arms for baseline characteristics. The majority of both PAT and PATE participants were African American (95 and $97 \%$ ), single (91 and $95 \%$ ), receiving Medicaid (93 and $90 \%$ ), overweight/obese prior to pregnancy (63 and $72 \%)$, and started their prenatal care in their second month of pregnancy (56 \% for both). Additionally, PAT and PATE participants were young (mean age $=23 \pm$ 4.6 years and $23 \pm 4.7$ years) and early in their second trimester of pregnancy (mean gestational age $=17 \pm$ 1.9 weeks and $18 \pm 2.4$ weeks). There were no significant differences between PAT and PATE participants at baseline. However, women that were retained throughout the gestational period were significantly more likely to have access to a motor vehicle as compared to those not retained ( $95 \%$ vs. $78 \%, p=0.036$; data not shown in the table). 
Table 1 Delta Healthy Sprouts participant baseline (early second trimester of pregnancy) demographic characteristics by treatment arm

\begin{tabular}{|c|c|c|c|c|c|}
\hline \multirow[b]{2}{*}{ Characteristic } & \multicolumn{2}{|c|}{$\operatorname{PAT}(n=43)$} & \multicolumn{2}{|c|}{ PATE $(n=39)$} & \multirow[b]{2}{*}{$P$} \\
\hline & $\mathrm{n}$ & $\%$ & $\mathrm{n}$ & $\%$ & \\
\hline Race & & & & & 1.000 \\
\hline African American & 41 & 95.3 & 38 & 97.4 & \\
\hline White & 2 & 4.7 & 1 & 2.6 & \\
\hline Marital status & & & & & 0.678 \\
\hline Single ${ }^{a}$ & 39 & 90.7 & 37 & 94.9 & \\
\hline Married & 4 & 9.3 & 2 & 5.1 & \\
\hline Education level & & & & & 0.684 \\
\hline 9th-11th grade & 7 & 16.3 & 7 & 17.9 & \\
\hline High school/GED & 15 & 34.9 & 12 & 30.8 & \\
\hline Some college/technical & 17 & 39.5 & 13 & 33.3 & \\
\hline College degree & 4 & 9.3 & 7 & 17.9 & \\
\hline Employment status & & & & & 0.468 \\
\hline Full time/part-time & 13 & 30.2 & 16 & 41.0 & \\
\hline Unemployed (looking) & 21 & 48.8 & 14 & 35.9 & \\
\hline Homemaker/student & 9 & 20.9 & 9 & 23.1 & \\
\hline Household monthly income ${ }^{b}$ & & & & & 0.284 \\
\hline$<\$ 500$ & 10 & 23.3 & 5 & 12.8 & \\
\hline$\$ 500-\$ 1000$ & 10 & 23.3 & 9 & 23.1 & \\
\hline$\$ 1001-\$ 1500$ & 4 & 9.3 & 12 & 30.8 & \\
\hline$\$ 1501-\$ 2000$ & 7 & 16.3 & 5 & 12.8 & \\
\hline$\$ 2001-\$ 4000$ & 6 & 14.0 & 3 & 7.7 & \\
\hline Don't know/refused & 6 & 14.0 & 5 & 12.8 & \\
\hline Smoker in household & 12 & 27.9 & 12 & 30.8 & 0.776 \\
\hline Smoker ${ }^{c}$ & & & & & 0.112 \\
\hline Current & 3 & 7.0 & 1 & 2.6 & \\
\hline Stopped before pregnancy & 1 & 2.3 & 0 & 0.0 & \\
\hline Stopped after became pregnant & 2 & 4.7 & 0 & 0.0 & \\
\hline No & 37 & 86.0 & 38 & 97.4 & \\
\hline Medicaid health insurance & 40 & 93.0 & 35 & 89.7 & 0.703 \\
\hline Receiving SNAP & 35 & 81.4 & 27 & 69.2 & 0.200 \\
\hline Receiving WIC & 38 & 88.4 & 31 & 79.5 & 0.271 \\
\hline Own/access to vehicle & 39 & 90.7 & 35 & 89.7 & 1.000 \\
\hline Receiving prenatal care & 43 & 100.0 & 39 & 100.0 & \\
\hline Pre-pregnancy weight class ${ }^{d}$ & & & & & 0.386 \\
\hline Underweight (BMI < 18.5) & 4 & 9.3 & 3 & 7.7 & \\
\hline Healthy weight $(18.5 \leq \mathrm{BMI}<25)$ & 12 & 27.9 & 8 & 20.5 & \\
\hline Overweight $(25 \leq \mathrm{BMI}<30)$ & 9 & 20.9 & 10 & 25.6 & \\
\hline \multirow[t]{2}{*}{ Obese (BMI $\geq 30)$} & 18 & 41.9 & 18 & 46.2 & \\
\hline & Mean & SD & Mean & SD & $P$ \\
\hline Age (years) & 23.3 & 4.58 & 22.7 & 4.69 & 0.537 \\
\hline Household size & 3.8 & 1.62 & 4.1 & 1.78 & 0.406 \\
\hline
\end{tabular}

Table 1 Delta Healthy Sprouts participant baseline (early second trimester of pregnancy) demographic characteristics by treatment arm (Continued)

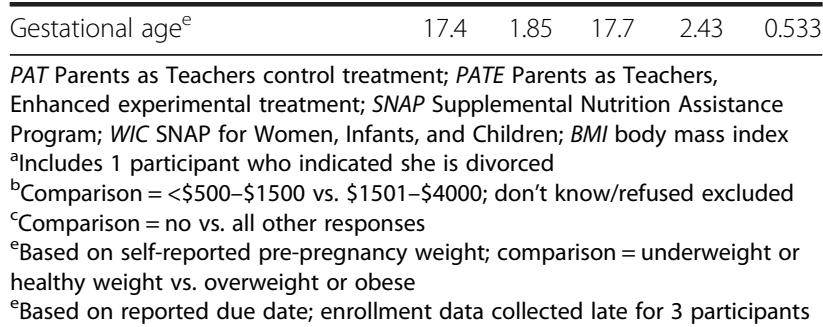

\section{Diet quality and nutrient intake outcomes}

Table 2 presents the results of the diet quality analyses by treatment arm and gestational visit. HEI-2010 total diet quality mean scores for PAT participants at GM 4, GM 6, and GM 8 were 41, 41, and 43 points, respectively; corresponding scores for PATE participants were 46,44 , and 45 points, respectively, reflecting overall poor diet quality for all participants at all gestational time points. In particular, HEI-2010 component mean scores were low for greens and beans, whole grains, seafood and plant protein foods, and sodium for both treatment arms. Significant treatment, time and interaction effects were not found for the HEI-2010 total or component scores.

Table 3 presents the results of the nutrient intake analyses by treatment arm and gestational visit. Significant treatment, time and interaction effects were not found for any of the nutrients modeled. However, there was a clinically meaningful reduction in the percentage of total energy from saturated fat in the PATE arm between GM 4 and GM 8 (10.6 to $9.6 \%)$. An unexpected but clinically meaningful reduction in mean calcium intake in the PATE arm between GM 4 and GM 8 (861 $\mathrm{mg}$ to $792 \mathrm{mg}$ ) also was observed.

Table 4 presents the results of the proportions of participants meeting nutrient intake recommendations by treatment arm and gestational visit. At baseline < $20 \%$ of women met intake recommendations for dietary fiber, sodium, or choline. Whereas, more than half of the women met intake recommendations for cholesterol, folate, iron, vitamin C, and vitamin D at baseline. Significant treatment, time and interaction effects were not found for any of the nutrients modeled. However, clinically meaningful increases in percentages of participants meeting recommendations for saturated fat intake between GM 4 and GM 8 were observed (PAT 30 to $42 \%$; PATE 47 to $64 \%$ ). Likewise, clinically meaningful increases in percentages of PAT participants meeting recommendations for cholesterol and vitamin $\mathrm{C}$ intakes between GM 4 and GM 8 were observed (65 to $84 \%$ and 86 to $97 \%$, respectively). An unanticipated albeit clinically meaningful decrease in percentage of PATE 
Table 2 Diet quality scores for Delta Healthy Sprouts participants in the gestational period by treatment arm and visit (time)

\begin{tabular}{|c|c|c|c|c|c|c|c|c|c|c|c|c|c|}
\hline \multirow{3}{*}{$\frac{\text { HEI-2010 Component }}{\text { Total vegetables }}$} & \multirow{3}{*}{$\begin{array}{l}\text { Arm } \\
\text { PAT }\end{array}$} & \multicolumn{3}{|c|}{ GM $4(n=43 \text { and } 38)^{a}$} & \multicolumn{3}{|c|}{ GM $6(n=39$ and 25$)$} & \multicolumn{3}{|c|}{ GM $8(n=36 \text { and } 22)^{b}$} & \multicolumn{3}{|l|}{$P$} \\
\hline & & \multirow{2}{*}{$\begin{array}{l}\text { LSM } \\
2.3\end{array}$} & \multicolumn{2}{|c|}{$95 \% \mathrm{Cl}$} & \multirow{2}{*}{$\begin{array}{l}\text { LSM } \\
1.8\end{array}$} & \multicolumn{2}{|c|}{$95 \% \mathrm{Cl}^{\mathrm{C}}$} & \multirow{2}{*}{$\begin{array}{l}\mathrm{LSM} \\
2.1\end{array}$} & \multicolumn{2}{|c|}{$95 \% \mathrm{Cl}^{\mathrm{C}}$} & \multirow{2}{*}{$\begin{array}{l}\text { Time } \\
0.877\end{array}$} & \multirow{2}{*}{$\begin{array}{l}\text { Arm } \\
0.237\end{array}$} & \multirow{2}{*}{$\begin{array}{ll}\text { Int } \\
0.367\end{array}$} \\
\hline & & & 1.8 & 2.7 & & 1.3 & 2.2 & & 1.6 & 2.5 & & & \\
\hline (Range 0-5) & PATE & 1.7 & 1.3 & 2.2 & 1.9 & 1.4 & 2.5 & 1.8 & 1.2 & 2.4 & & & \\
\hline Greens \& beans & PAT & 0.6 & 0.1 & 1.0 & 0.8 & 0.3 & 1.3 & 0.5 & 0.0 & 1.0 & 0.243 & 0.820 & 0.797 \\
\hline (Range 0-5) & PATE & 0.6 & 0.1 & 1.1 & 0.8 & 0.2 & 1.5 & 0.2 & 0.0 & 0.9 & & & \\
\hline Total fruit & PAT & 1.3 & 0.7 & 1.9 & 2.2 & 1.5 & 2.8 & 1.5 & 0.8 & 2.1 & 0.713 & 0.860 & 0.086 \\
\hline (Range 0-5) & PATE & 1.8 & 1.1 & 2.4 & 1.4 & 0.6 & 2.2 & 1.9 & 1.1 & 2.8 & & & \\
\hline Whole fruit & PAT & 1.1 & 0.4 & 1.7 & 1.8 & 1.1 & 2.4 & 1.2 & 0.5 & 1.8 & 0.248 & 0.214 & 0.539 \\
\hline (Range 0-5) & PATE & 1.6 & 0.9 & 2.2 & 1.8 & 1.0 & 2.6 & 1.9 & 1.1 & 2.8 & & & \\
\hline Whole grains & PAT & 2.4 & 1.4 & 3.4 & 1.8 & 0.7 & 2.8 & 2.1 & 1.1 & 3.2 & 0.818 & 0.388 & 0.375 \\
\hline (Range 0-10) & PATE & 2.5 & 1.4 & 3.5 & 3.1 & 1.8 & 4.4 & 2.1 & 0.7 & 3.5 & & & \\
\hline Dairy & PAT & 4.4 & 3.5 & 5.2 & 3.6 & 2.7 & 4.5 & 4.0 & 3.1 & 4.9 & 0.311 & 0.086 & 0.543 \\
\hline (Range 0-10) & PATE & 3.7 & 2.7 & 4.6 & 3.4 & 2.3 & 4.5 & 2.7 & 1.5 & 3.9 & & & \\
\hline Total protein foods & PAT & 4.4 & 4.0 & 4.8 & 4.3 & 3.9 & 4.7 & 4.2 & 3.8 & 4.6 & 0.960 & 0.561 & 0.548 \\
\hline (Range 0-5) & PATE & 4.3 & 3.9 & 4.7 & 4.5 & 4.0 & 4.9 & 4.6 & 4.0 & 5.1 & & & \\
\hline Seafood \& plant proteins & PAT & 0.7 & 0.1 & 1.3 & 1.0 & 0.4 & 1.6 & 0.8 & 0.2 & 1.4 & 0.960 & 0.813 & 0.362 \\
\hline (Range 0-5) & PATE & 1.2 & 0.6 & 1.8 & 0.7 & 0.0 & 1.4 & 0.9 & 0.1 & 1.7 & & & \\
\hline Fatty acids & PAT & 5.2 & 4.1 & 6.2 & 5.2 & 4.2 & 6.3 & 6.4 & 5.2 & 7.5 & 0.397 & 0.060 & 0.576 \\
\hline (Range 0-10) & PATE & 6.8 & 5.7 & 7.9 & 6.3 & 5.0 & 7.7 & 6.8 & 5.3 & 8.2 & & & \\
\hline Sodium & PAT & 2.4 & 1.4 & 3.3 & 2.9 & 2.0 & 3.9 & 2.9 & 1.9 & 3.9 & 0.780 & 0.118 & 0.653 \\
\hline (Range 0-10) & PATE & 3.5 & 2.5 & 4.5 & 3.1 & 1.9 & 4.4 & 3.7 & 2.4 & 5.0 & & & \\
\hline Refined grains & PAT & 5.8 & 4.8 & 6.9 & 4.5 & 3.4 & 5.6 & 5.3 & 4.2 & 6.5 & 0.054 & 0.104 & 0.554 \\
\hline (Range 0-10) & PATE & 6.2 & 5.1 & 7.3 & 5.0 & 3.6 & 6.4 & 7.0 & 5.5 & 8.5 & & & \\
\hline Empty calories & PAT & 10.5 & 8.8 & 12.2 & 10.7 & 9.0 & 12.5 & 12.2 & 10.4 & 14.1 & 0.759 & 0.450 & 0.519 \\
\hline (Range 0-20) & PATE & 11.9 & 10.1 & 13.7 & 11.9 & 9.7 & 14.1 & 11.5 & 9.2 & 13.9 & & & \\
\hline Total & PAT & 40.9 & 37.1 & 44.8 & 40.6 & 36.5 & 44.6 & 43.2 & 39.0 & 47.5 & 0.696 & 0.100 & 0.813 \\
\hline (Range 0-100) & PATE & 45.6 & 41.5 & 49.7 & 43.9 & 38.9 & 49.0 & 45.1 & 39.7 & 50.5 & & & \\
\hline
\end{tabular}

GM gestational month; HEl-2010 Healthy Eating Index-2010; LSM least squares mean; CI confidence interval; Int interaction (time $\mathrm{x}$ arm); PAT Parents as Teachers control treatment; PATE Parents as Teachers Enhanced experimental treatment ${ }^{\mathrm{a}}$ Missed dietary recall for 1 PATE participant

b Excluded dietary recall for 1 PATE participant (in hospital and consumed ice only)

${ }^{c}$ Negative lower limits are not feasible and were changed to 0

participants meeting recommendations for calcium intake between GM 4 and GM 8 also was observed (29 to $13 \%)$.

\section{Discussion}

In this paper, we describe and compare the gestational dietary intake outcomes of rural, Southern, primarily African American pregnant women participating in the Delta Healthy Sprouts randomized, comparative impact trial. Overall, our results suggest that the PAT curriculum and the more complex PATE intervention, were not effective at improving maternal diet quality or the adequacy of intake for several nutrients including dietary fiber, calcium and choline during the gestational period.

Other lifestyle interventions conducted in the gestational period have been successful at improving maternal diet quality and nutrient intake. The UPBEAT trial [38], conducted in the United Kingdom with a largely Caucasian population, was effective at improving diet quality in pregnant women with obesity. This one-on-one intervention was delivered in an antenatal clinic once weekly for 8 weeks with a health educator beginning between 16 and 19 weeks gestation. The intervention was successful at reducing maternal glycemic load and total and saturated fat intake and increasing dietary fiber. The LIMIT trial [18], a randomized prenatal dietary and lifestyle intervention conducted in a cohort of pregnant overweight and obese, predominately Caucasian, high SES, Australian women, was effective at improving maternal diet quality, measured by HEI, and intake of dietary fiber and saturated fat compared to standard care [18]. This intervention included six sessions implemented on 
Table 3 Nutrient intakes for Delta Healthy Sprouts participants in the gestational period by treatment arm and visit (time)

\begin{tabular}{|c|c|c|c|c|c|c|c|c|c|c|c|c|c|}
\hline \multirow{3}{*}{$\frac{\text { Nutrient }}{\text { Total fat (\% energy) }}$} & \multirow{3}{*}{$\begin{array}{l}\text { Arm } \\
\text { PAT }\end{array}$} & \multicolumn{3}{|c|}{ GM $4(n=43 \text { and } 38)^{a}$} & \multicolumn{3}{|c|}{ GM $6(n=39$ and 25$)$} & \multicolumn{3}{|c|}{$\mathrm{GM} 8(n=36 \text { and } 22)^{\mathrm{b}}$} & \multicolumn{3}{|l|}{$P$} \\
\hline & & \multirow{2}{*}{$\begin{array}{l}\text { LSM } \\
35.7\end{array}$} & \multicolumn{2}{|c|}{$95 \% \mathrm{Cl}$} & \multirow{2}{*}{$\begin{array}{l}\text { LSM } \\
32.8\end{array}$} & \multicolumn{2}{|c|}{$95 \% \mathrm{Cl}$} & \multirow{2}{*}{$\frac{\mathrm{LSM}}{34.6}$} & \multicolumn{2}{|c|}{$95 \% \mathrm{Cl}$} & \multirow{2}{*}{$\begin{array}{l}\text { Time } \\
0.175\end{array}$} & \multirow{2}{*}{$\frac{\text { Arm }}{0.990}$} & \multirow{2}{*}{$\frac{\ln t}{0.233}$} \\
\hline & & & 33.4 & 38.0 & & 30.5 & 35.2 & & 32.1 & 37.1 & & & \\
\hline $\mathrm{RDI} \leq 35 \%$ & PATE & 35.6 & 33.2 & 38.1 & 34.9 & 32.0 & 37.8 & 32.7 & 29.5 & 35.8 & & & \\
\hline Saturated fat (\% energy) & PAT & 11.4 & 10.4 & 12.5 & 10.7 & 9.7 & 11.7 & 10.5 & 9.5 & 11.6 & 0.204 & 0.178 & 0.780 \\
\hline $\mathrm{RDI} \leq 10 \%$ & PATE & 10.6 & 9.6 & 11.6 & 10.4 & 9.3 & 11.7 & 9.6 & 8.5 & 10.8 & & & \\
\hline Cholesterol $(\mathrm{mg})^{c}$ & PAT & 203 & 141 & 266 & 193 & 130 & 256 & 165 & 102 & 227 & 0.211 & 0.151 & 0.143 \\
\hline $\mathrm{RDI} \leq 300 \mathrm{mg}$ & PATE & 179 & 116 & 242 & 248 & 185 & 311 & 228 & 166 & 291 & & & \\
\hline Fiber $(g)^{c}$ & PAT & 10.7 & 8.4 & 13.1 & 11.7 & 9.4 & 14.0 & 11.3 & 9.0 & 13.6 & 0.881 & 0.607 & 0.869 \\
\hline $\mathrm{RDI} \geq 28 \mathrm{~g}$ & PATE & 10.2 & 7.9 & 12.5 & 11.3 & 9.0 & 13.6 & 10.4 & 8.1 & 12.7 & & & \\
\hline Sodium (mg) & PAT & 3770 & 3332 & 4208 & 3839 & 3379 & 4298 & 3725 & 3246 & 4203 & 0.700 & 0.334 & 0.900 \\
\hline $\mathrm{RDI} \leq 1500 \mathrm{mg}$ & PATE & 3624 & 3158 & 4090 & 3718 & 3144 & 4293 & 3378 & 2766 & 3990 & & & \\
\hline Calcium (mg) ${ }^{d}$ & PAT & 960 & 842 & 1094 & 939 & 818 & 1078 & 966 & 837 & 1115 & 0.305 & 0.281 & 0.151 \\
\hline $\mathrm{RDI}=1000 \mathrm{mg}$ & PATE & 861 & 749 & 990 & 1015 & 855 & 1205 & 792 & 659 & 951 & & & \\
\hline Folate (mcg) & PAT & 1246 & 1071 & 1421 & 1275 & 1092 & 1459 & 1252 & 1061 & 1444 & 0.904 & 0.278 & 0.990 \\
\hline $\mathrm{RDI}=600 \mathrm{mcg}$ & PATE & 1142 & 956 & 1329 & 1200 & 970 & 1429 & 1157 & 912 & 1401 & & & \\
\hline $\operatorname{Iron}(\mathrm{mg})^{c}$ & PAT & 41.2 & 36.3 & 46.2 & 41.6 & 36.7 & 46.5 & 41.9 & 37.0 & 46.9 & 0.710 & 0.555 & 0.917 \\
\hline $\mathrm{RDI}=27 \mathrm{mg}$ & PATE & 39.2 & 34.2 & 44.1 & 43.0 & 38.1 & 47.9 & 40.3 & 35.4 & 45.2 & & & \\
\hline Vitamin C $(\mathrm{mg})^{c, e}$ & PAT & 162 & 117 & 207 & 197 & 152 & 241 & 183 & 139 & 228 & 0.690 & 0.471 & 0.947 \\
\hline $\mathrm{RDI}=85 \mathrm{mg}$ & PATE & 153 & 109 & 198 & 179 & 134 & 224 & 163 & 118 & 208 & & & \\
\hline Vitamin D (mcg) & PAT & 12.4 & 10.7 & 14.2 & 11.6 & 9.8 & 13.4 & 12.3 & 10.5 & 14.2 & 0.907 & 0.380 & 0.559 \\
\hline $\mathrm{RDI}=5 \mathrm{mcg}$ & PATE & 10.9 & 9.1 & 12.8 & 11.3 & 9.2 & 13.4 & 11.0 & 8.8 & 13.2 & & & \\
\hline Choline $(\mathrm{mg})^{c}$ & PAT & 240 & 181 & 299 & 248 & 189 & 307 & 206 & 147 & 265 & 0.745 & 0.392 & 0.403 \\
\hline $\mathrm{RDI}=450 \mathrm{mg}$ & PATE & 223 & 164 & 282 & 280 & 221 & 339 & 239 & 180 & 298 & & & \\
\hline
\end{tabular}

GM gestational month; HEl-2010 Healthy Eating Index-2010; LSM least squares mean; Cl confidence interval; Int interaction (time $\mathrm{x}$ arm); PAT Parents as Teachers control treatment; PATE Parents as Teachers Enhanced experimental treatment

${ }^{a}$ Missed dietary recall for 1 PATE participant

${ }^{b}$ Excluded dietary recall for 1 PATE participant (in hospital and consumed ice only)

'Values are medians and associated $95 \%$ confidence intervals from quantile regression

${ }^{\mathrm{d}} \mathrm{RDI}=1300 \mathrm{mg}$ for participants 18 years of age

${ }^{e} \mathrm{RDI}=80 \mathrm{mg}$ for participants 18 years of age

an individual basis both in person and over the phone by a registered dietitian and trained research assistants. While our one-on-one intervention was implemented monthly between GM 4 and 9 in the participant's home by a trained lay parent educator, only three of the five lessons (GM 5, 6 and 7) were delivered prior to the final gestational diet intake assessment at GM 8. It may be that the dose of our intervention was too low to foster or the timing of our final diet intake was not optimal for detecting positive dietary changes. Lifestyle changes, including dietary changes, involve the adoption of new positive behaviors [39] and research suggests that lifestyle habits can take anywhere from 66 to 254 days to form [40]. Thus, the short duration of our gestational intervention may not have allowed for the time needed to develop and adopt positive dietary behaviors [39]. Given that our intervention spans into the postpartum period, future analyses will assess the adoption of positive diet behaviors in this cohort of women over a longer period of time.
The UPBEAT [38] and LIMIT [18] prenatal lifestyle intervention trials focused their messaging solely on maternal gestational weight gain, diet, and lifestyle behaviors. The Delta Healthy Sprouts Project was built on the framework of the evidence-based PAT curriculum which covers topics related to parental knowledge of child development, positive parenting practices, early detection of developmental delays, preventing child abuse, and school readiness. Thus, combining the PATE diet and lifestyle curriculum with the existing PAT program may have diluted the diet and lifestyle messaging. Further, some studies suggest that multi-component dietary advice such as USDA MyPlate, which was used as the framework for the PATE dietary messaging, can be overwhelming to some individuals due to the knowledge required to comprehend the complex recommendations [41]. The considerable and diverse number of messages provided in the PATE curriculum may have overwhelmed the women randomized to this arm of the 
Table 4 Percentages of Delta Healthy Sprouts participants meeting recommended nutrient intakes in the gestational period by treatment arm and visit (time)

\begin{tabular}{|c|c|c|c|c|c|c|c|c|c|c|c|c|c|}
\hline \multirow{3}{*}{$\frac{\text { Nutrient }^{c}}{\text { Total fat (\% energy) }}$} & \multirow{3}{*}{$\begin{array}{l}\text { Arm } \\
\text { PAT }\end{array}$} & \multicolumn{3}{|c|}{ GM $4(n=43 \text { and } 38)^{a}$} & \multicolumn{3}{|c|}{ GM 6 ( $n=39$ and 25) } & \multicolumn{3}{|c|}{ GM $8(n=36 \text { and } 22)^{\mathrm{b}}$} & \multicolumn{3}{|l|}{$P$} \\
\hline & & \multirow{2}{*}{$\begin{array}{l}\text { LSM } \\
48.8\end{array}$} & \multicolumn{2}{|c|}{$95 \% \mathrm{Cl}$} & \multirow{2}{*}{$\begin{array}{l}\text { LSM } \\
53.9\end{array}$} & \multicolumn{2}{|c|}{$95 \% \mathrm{Cl}$} & \multirow{2}{*}{$\begin{array}{l}\text { LSM } \\
49.7\end{array}$} & \multicolumn{2}{|c|}{$95 \% \mathrm{Cl}$} & \multirow{2}{*}{$\begin{array}{l}\text { Time } \\
0.650\end{array}$} & \multirow{2}{*}{$\begin{array}{l}\text { Arm } \\
0.467\end{array}$} & \multirow{2}{*}{$\frac{\operatorname{lnt}}{0.684}$} \\
\hline & & & 34.1 & 63.8 & & 38.0 & 69.0 & & 33.6 & 65.9 & & & \\
\hline $\mathrm{RDA} \leq 35 \%$ & PATE & 50.0 & 34.2 & 65.8 & 55.4 & 35.8 & 73.6 & 64.5 & 42.6 & 81.6 & & & \\
\hline Saturated fat (\% energy) & PAT & 30.2 & 18.2 & 45.8 & 43.8 & 29.0 & 59.9 & 41.6 & 26.5 & 58.4 & 0.285 & 0.063 & 0.570 \\
\hline $\mathrm{RDA} \leq 10 \%$ & PATE & 47.4 & 31.9 & 63.4 & 48.2 & 29.5 & 67.5 & 63.5 & 41.7 & 80.9 & & & \\
\hline Cholesterol (mg) & PAT & 65.1 & 49.5 & 78.1 & 64.3 & 47.9 & 77.9 & 83.7 & 67.5 & 92.7 & 0.414 & 0.607 & 0.304 \\
\hline $\mathrm{RDA} \leq 300 \mathrm{mg}$ & PATE & 68.4 & 51.8 & 81.4 & 69.1 & 48.5 & 84.2 & 67.1 & 45.1 & 83.6 & & & \\
\hline Fiber (g) & PAT & 0.0 & NC & NC & 0.0 & NC & NC & 0.0 & NC & $\mathrm{NC}$ & NC & NC & NC \\
\hline $\mathrm{RDA} \geq 28 \mathrm{~g}$ & PATE & 7.9 & NC & $\mathrm{NC}$ & 0.0 & NC & NC & 9.1 & NC & $\mathrm{NC}$ & & & \\
\hline Sodium (mg) & PAT & 0.0 & $\mathrm{NC}$ & $\mathrm{NC}$ & 2.6 & NC & NC & 5.6 & NC & NC & NC & NC & NC \\
\hline $\mathrm{RDA} \leq 1500 \mathrm{mg}$ & PATE & 15.8 & $\mathrm{NC}$ & $\mathrm{NC}$ & 0.0 & NC & NC & 0.0 & NC & $\mathrm{NC}$ & & & \\
\hline Calcium (mg) ${ }^{d}$ & PAT & 41.9 & 27.9 & 57.3 & 40.7 & 26.3 & 57.0 & 44.3 & 28.8 & 61.0 & 0.245 & 0.054 & 0.130 \\
\hline $\mathrm{RDA}=1000 \mathrm{mg}$ & PATE & 28.9 & 16.5 & 45.6 & 41.8 & 24.2 & 61.8 & 13.4 & 4.2 & 35.2 & & & \\
\hline Folate (mcg) & PAT & 90.7 & 77.4 & 96.5 & 91.4 & 77.6 & 97.0 & 88.7 & 73.8 & 95.6 & 0.929 & 0.486 & 0.871 \\
\hline $\mathrm{RDA}=600 \mathrm{mcg}$ & PATE & 86.8 & 71.8 & 94.5 & 85.1 & 66.9 & 94.1 & 85.7 & 65.5 & 95.0 & & & \\
\hline Iron (mg) & PAT & 90.7 & 77.4 & 96.5 & 91.1 & 77.5 & 96.8 & 88.4 & 73.8 & 95.4 & 0.975 & 0.260 & 0.486 \\
\hline $\mathrm{RDA}=27 \mathrm{mg}$ & PATE & 81.6 & 65.8 & 91.1 & 79.2 & 61.4 & 90.2 & 84.9 & 65.7 & 94.3 & & & \\
\hline Vitamin $C(\mathrm{mg})^{\mathrm{e}}$ & PAT & 86.0 & 71.8 & 93.7 & 89.4 & 74.9 & 96.0 & 97.3 & 82.3 & 99.6 & 0.267 & 0.184 & 0.495 \\
\hline $\mathrm{RDA}=85 \mathrm{mg}$ & PATE & 84.2 & 68.6 & 92.9 & 81.4 & 61.6 & 92.3 & 87.0 & 65.6 & 95.9 & & & \\
\hline Vitamin D (mcg) & PAT & 83.7 & 69.3 & 92.2 & 85.0 & 70.2 & 93.2 & 82.6 & 66.8 & 91.8 & 0.949 & 0.699 & 0.790 \\
\hline $\mathrm{RDA}=5 \mathrm{mcg}$ & PATE & 78.9 & 62.9 & 89.3 & 79.9 & 61.4 & 90.9 & 83.4 & 63.2 & 93.6 & & & \\
\hline Choline (mg) & PAT & 14.0 & 6.2 & 28.3 & 15.7 & 7.2 & 31.1 & 8.1 & 2.5 & 23.2 & 0.525 & 0.992 & 0.349 \\
\hline $\mathrm{RDA}=450 \mathrm{mg}$ & PATE & 18.4 & 8.8 & 34.5 & 7.5 & 1.8 & 26.7 & 13.0 & 4.0 & 34.7 & & & \\
\hline
\end{tabular}

GM gestational month; HEl-2010 Healthy Eating Index-2010; LSM least squares mean; Cl, confidence interval; Int interaction (time $\mathrm{x}$ arm); PAT Parents as Teachers control treatment; PATE Parents as Teachers Enhanced experimental treatment; NC, not computed since model failed to converge due to time points with zero and close to zero percentages

${ }^{a}$ Missed dietary recall for 1 PATE participant

${ }^{b}$ Excluded dietary recall for 1 PATE participant (in hospital and consumed ice only)

'Based on food and supplements combined

${ }^{\mathrm{d}} \mathrm{RDA}=1300 \mathrm{mg}$ for participants 18 years of age

${ }^{\mathrm{e}} \mathrm{RDA}=80 \mathrm{mg}$ for participants 18 years of age

study. The higher attrition and lower compliance with home visits during the gestational period, despite no other notable participant characteristic differences, suggests some level of displeasure with the PATE lessons. The standard PAT curriculum provides some simple maternal dietary advice including importance of taking prenatal vitamins, safe and healthy fish options during pregnancy, and consuming more calories during the $2^{\text {nd }}$ and $3^{\text {rd }}$ trimester. The three point increase in total HEI2010 score and improvements in saturated fat, cholesterol and vitamin $C$ intake in the PAT arm suggests that simple nutrition messaging, when presented in the context of a more global maternal and child health curriculum, may promote positive maternal dietary behaviors in the gestational period. Further research is needed to better understand the impact of simple vs. complex nutrition messaging on maternal diet in rural, disadvantaged pregnant women.

Women are bombarded with information during pregnancy [39]. All of the women in our study were receiving prenatal care at baseline and more than $80 \%$ of our participants were enrolled in the Women Infants and Children (WIC) supplemental nutrition program. Further, 88 and $83 \%$, respectively, of our PAT and PATE participants indicated they had received advice about healthy eating from their prenatal care provider. Thus, it is probable that participants were overwhelmed by a vast amount of health and lifestyle-related information and possible that our diet advice conflicted with advice received from their clinician or WIC counselor. Research suggests that pregnant women tend to place the most importance on recommendations made by their doctor/ 
clinical provider [42, 43]. Whereas women tend to be somewhat dismissive of health recommendations that were not addressed by their health care provider. Therefore, women in the PATE arm may have been less likely to follow our diet recommendations if similar advice was not also encouraged by their provider [39]. Future interventions should consider involving obstetrical healthcare providers to allow for consistent messaging around gestational lifestyle behaviors.

Our study was implemented in the largely rural, socioeconomically disadvantaged LMD region. Studies suggest that socioeconomic status (SES) is positively associated with diet quality [44]. There are several hypothesized explanations for this relationship. Research suggests that low SES individuals may experience greater reward from food given their limited access to non-food related rewards due to cost or access [45]. Further, low SES neighborhoods and regions, like the LMD, have fewer large supermarkets and more convenience stores and fast-food outlets that offer relatively low cost, nutrient poor, energy dense foods [46]. Research has shown that living in low SES areas is associated with less consumption of fruits and vegetables and a tendency to select foods that are more readily accessible [47, 48]. Epstein and colleagues [45] suggest that even when access to healthier food is improved through participation in food assistance programs, low SES individuals will continue to choose foods of low nutritive value that provide greater food reinforcement. Epstein hypothesizes that reducing access to low quality foods through policy-related changes and increasing the availability of low-cost, non-food reinforcement, may encourage the selection of healthier food choices in low SES populations.

Several strengths of the Delta Healthy Sprouts Project deserve consideration. Participants were visited in their homes, thus alleviating the burden of travel for these rural residents. Although the study's sample size is relatively small, no other studies were found which focused on the diet, including nutrient intake and diet quality, of this population of pregnant women. Dietary intake was measured three times in the gestational period (vs. the more typical pre/post measurement schedule). Despite the strengths of this study, there are some limitations that bear mentioning. The potential for socially desirable responses for survey questions and dietary recalls cannot be discounted. To reduce this bias potential, parent educators were trained to not ask leading questions and to maintain neutral facial expressions, particularly when conducting dietary recalls. Data collection was not blinded and therefore a potential source of bias. However, because the data was collected in the participants' homes, it was not practically, logistically, or financially feasible to have a second set of blinded research staff whose purpose was solely to collect data. Stopping recruitment before target enrollment numbers were reached likely limited our ability to detect statistically significant differences between treatment arms, especially given the low compliance rates with visits observed in the PATE arm (Thomson JL, under review). A post hoc power and sample size analysis revealed that the actual sample sizes of 33 PAT and 26 PATE participants were sufficient to detect a statistically significant difference of 9.9 points in change in total HEI-2010 scores between treatment arms with $80 \%$ power and a type 1 error rate of 0.05 . Given the relatively short duration of the intervention in the gestational period, achieving such a large effect was unlikely. Finally, the high attrition rate may limit generalizability of these study results.

\section{Conclusion}

In conclusion, designing effective interventions that enable women to improve their dietary intake during pregnancy remains a challenge as evidenced by the lack of impact on maternal diet quality observed in the current study. Future interventions designed for rural, low income, minority populations of pregnant women might consider recruiting women earlier in their pregnancy (e.g., first trimester), increasing the treatment dosage, and involving prenatal care providers to ensure reinforcement and consistency in health behavior messaging. Given the importance of optimum maternal nutrition for positive maternal and fetal health outcomes, research efforts need to remain focused on populations at risk for poor pregnancy and birth outcomes, including residents of the LMD region.

\section{Abbreviations}

BMI, body mass index; DGAs, dietary guidelines for Americans; DPP, Diabetes Prevention Program; GM, gestational month; HEl, healthy eating index; InFANT, Infant Feeding and Nutrition Trial; LMD, Lower Mississippi Delta; NDSR, nutrition data system for research; PAT, parents as teachers; PATE, Parents as Teachers enhanced curriculum; US, United States; USDA, United States Department of Agriculture; WIC, Women, infants, and children

\section{Acknowledgements \\ We thank Debra Johnson, Shakari Moore, and Donna Ransome for their research support, and the mothers who participated in this study. The views expressed are solely those of the authors and do not reflect the official policy or position of the US government.}

\section{Funding}

This research is supported by the United States Department of Agriculture, Agricultural Research Service Project 6001-53000-001-00D (Thomson), and in kind support from the Delta Health Alliance.

Availability of data and materials

De-identified data supporting our findings may be made available upon request and as deemed appropriate to Jessica Thomson at jessica.thomson@ars.usda.gov. The Delta Healthy Sprouts curriculum is available at: http://www.ars.usda.gov/ Main/docs.htm?docid=25157. 


\section{Authors' contributions}

LMT-H contributed to the study design, data analysis and interpretation, and drafted the manuscript. JLT contributed to the study design; data acquisition, analysis, and interpretation. MHG contributed to the data acquisition, analysis, and interpretation. SO contributed to the data acquisition, analysis, and interpretation. All authors participated in the drafting and revising of the manuscript. All authors read and approved the final manuscript.

\section{Authors' information}

No additional information to disclose.

\section{Competing interests}

The authors declare that they have no competing interests

\section{Consent for publication}

Not applicable.

\section{Ethics approval and consent to participate}

The project was approved by the Institutional Review Board of Delta State University, Cleveland MS (\#12-024) and all participants gave written informed consent prior to any study engagement.

\section{Author details}

Department of Medicine and Cancer Center, University of Illinois at Chicago, 1747 W Roosevelt Road, \#416, Chicago, IL 60618, USA. ${ }^{2}$ United States Department of Agriculture, Agricultural Research Service, 141 Experiment Station Road, Stoneville, MS 38776, USA.

Received: 12 May 2016 Accepted: 4 August 2016

Published online: 17 August 2016

\section{References}

1. Ramakrishnan U, Grant F, Goldenberg T, Zongrone A, Martorell R. Effect of women's nutrition before and during early pregnancy on maternal and infant outcomes: a systematic review. Paediatr Perinat Epidemiol. 2012;26 Suppl 1:285-301.

2. King JC. The risk of maternal nutritional depletion and poor outcomes increases in early or closely spaced pregnancies. J Nutr. 2003;133(5 Suppl 2): 1732S-6S.

3. Rifas-Shiman SL, Rich-Edwards JW, Kleinman KP, Oken E, Gillman MW. Dietary quality during pregnancy varies by maternal characteristics in Project Viva: a US cohort. J Am Diet Assoc. 2009;109(6):1004-11. Pubmed Central PMCID: 4098830

4. Poon AK, Yeung E, Boghossian N, Albert PS, Zhang C. Maternal Dietary Patterns during Third Trimester in Association with Birthweight Characteristics and Early Infant Growth. Scientifica. 2013;2013:786409. Pubmed Central PMCID: 3893866.

5. Rodriguez-Bernal CL, Rebagliato M, Chatzi L, Carbinell CC, Martos C, Ballester F. Maternal Diet Quality and Pregnancy Outcomes In: Preedy VR, Lan-Anh H, Patel V, B., editors. Diet Quality 1. 1 ed. New York, NY: Humana Press; 2013. p. 65-79.

6. Martin CL, Sotres-Alvarez D, Siega-Riz AM. Maternal Dietary Patterns during the Second Trimester Are Associated with Preterm Birth. J Nutr. 2015;145(8): 1857-64. Pubmed Central PMCID: 4516771.

7. Hu F. Dietary Pattern Analysis: A New Direction in Nutritional Epidemiology. Current Opinion in Lipidology 132002. 2002. p. 3-9.

8. Baskin R, Hill B, Jacka FN, O'Neil A, Skouteris H. Antenatal dietary patterns and depressive symptoms during pregnancy and early post-partum. Matern Child Nutr. 2016. doi:10.1111/mcn.12218. [Epub ahead of print].

9. Shin MK, Kim YS, Kim JH, Kim SH, Kim Y. Dietary Patterns and Their Associations with the Diet Quality Index-International (DQI-I) in Korean Women with Gestational Diabetes Mellitus. Clin Nutr Res. 2015;4(4):216-24. Pubmed Central PMCID: 4641983

10. Brantsaeter AL, Haugen M, Samuelsen SO, Torjusen H, Trogstad L, Alexander J, et al. A dietary pattern characterized by high intake of vegetables, fruits, and vegetable oils is associated with reduced risk of preeclampsia in nulliparous pregnant Norwegian women. J Nutr. 2009;139(6):1162-8. Pubmed Central PMCID: 2682988

11. USDA. Dietary Guidelines for Americans, 2010. In: Services HaH, editor. 7 ed. Washington, DC: U.S. Government Printing Office 2010.
12. Guenther PM, Casavale KO, Reedy J, Kirkpatrick SI, Hiza HAB, Kuczynski KJ, et al. Update of the Healthy Eating Index: HEl-2010. J Acad Nutr Diet. 2013; 113(4):569-80. PubMed PMID: WOS:000317257300013.

13. Yu DX, Sonderman J, Buchowski MS, McLaughlin JK, Shu XO, Steinwandel M, et al. Healthy Eating and Risks of Total and Cause-Specific Death among LowIncome Populations of African-Americans and Other Adults in the Southeastern United States: A Prospective Cohort Study. Plos Med. 2015;12(5). PubMed PMID: WOS:000355304100008.

14. Fogli-Cawley JJ, Dwyer JT, Saltzman E, McCullough ML, Troy LM, Meigs JB, et al. The 2005 Dietary Guidelines for Americans and risk of the metabolic syndrome. Am J Clin Nutr. 2007;86(4):1193-201. PubMed PMID: WOS: 000250134600042

15. Pick ME, Edwards M, Moreau D, Ryan EA. Assessment of diet quality in pregnant women using the healthy eating index. J Am Diet Assoc. 2005; 105(2):240-6. PubMed PMID: WOS:000226733100015.

16. Melere C, Hoffmann JF, Nunes MAA, Drehmer M, Buss C, Ozcariz SGl, et al. Healthy eating index for pregnancy: adaptation for use in pregnant women in Brazil. Rev Saude Publ. 2013;47(1):20-8. PubMed PMID: WOS:000320026700004.

17. Moran $\sqcup$, Sui Z, Cramp CS, Dodd JM. A decrease in diet quality occurs during pregnancy in overweight and obese women which is maintained post-partum. Int J Obesity. 2013;37(5):704-11. PubMed PMID: WOS:000318772400012.

18. Dodd JM, Cramp C, Sui ZX, Yelland LN, Deussen AR, Grivell RM, et al. The effects of antenatal dietary and lifestyle advice for women who are overweight or obese on maternal diet and physical activity: the LIMIT randomised trial. Bmc Med. 2014;13:12. PubMed PMID: WOS:000344122400001.

19. Robert Wood Johnson Foundation. County Health Rankings and Roadmaps, 2016 July 16, 2016. Available from: http://www.countyhealthrankings.org Accessed 16 July 2016

20. Mississippi State Department of Health. Vital Statistics Mississippi 2012 July 16, 2016. Available from: http://msdh.ms.gov/phs/2012/Bulletin/vr2012.pdf. Accessed 16 July 2016

21. Smith BL, Sandlin AT, Bird TM, Steelman SC, Magann EF. Maternal mortality in the Mississippi delta region. South Med J. 2014;107(5):275-9. doi:10.1097/ SMJ.0000000000000102.

22. Bhutta ZA, Das JK, Rizvi A. The Lancet Nutrition Interventions Review Group, and the Maternal and Child Nutrition Study Group. Evidence-based interventions for improvement of maternal and child nutrition: what can be done and at what cost? Lancet. 2013;382(9890):396. PubMed PMID: WOS:000322638500029.

23. Thomson JL, Tussing-Humphreys LM, Goodman MH, Olender S. Baseline Demographic. Psychosocial, and Behavioral Characteristics of Rural, Southern Women in Early Pregnancy. Maternal and child health journal: Anthropometric; 2016.

24. Thomson JL, Tussing-Humphreys LM, Goodman MH. Delta Healthy Sprouts: A randomized comparative effectiveness trial to promote maternal weight control and reduce childhood obesity in the Mississippi Delta. Contemp Clin Trials. 2014;38(1):82-91. PubMed PMID: WOS:000337203900009.

25. PAT. Parents as Teachers National Center Inc. [January 25, 2016]. Available from: http://www.parentsasteachers.org. Accessed 16 July 2016

26. Knowler WC, Barrett-Connor E, Fowler SE, Hamman RF, Lachin JM, Walker EA et al. Reduction in the incidence of type 2 diabetes with lifestyle intervention or metformin. N Engl J Med. 2002;346(6):393-403. Pubmed Central PMCID: 1370926.

27. Campbell KJ, Lioret S, McNaughton SA, Crawford DA, Salmon J, Ball K, et al. A parent-focused intervention to reduce infant obesity risk behaviors: a randomized trial. Pediatrics. 2013;131(4):652-60.

28. Nowak AJ, Casamassimo PS. Using anticipatory guidance to provide early dental intervention. J Am Dent Assoc. 1995;126(8):1156-63.

29. Mullis RL, Hill EW, Readdick CA. Attachment and social support among adolescents. J Genet Psychol. 1999;160(4):500-2.

30. Feskanich D, Sielaff BH, Chong K, Buzzard IM. Computerized collection and analysis of dietary intake information. Comput Methods Prog Biomed. 1989;30(1):47-57.

31. Harnack L, Stevens M, Van Heel N, Schakel S, Dwyer JT, Himes J. A computer-based approach for assessing dietary supplement use in conjunction with dietary recalls. J Food Composition Anal. 2008; 21(Suppliment 1):S78-82. Pubmed Central PMCID: 2151738.

32. Basiotis PP, Carlson A, Gerrior SA, Juan WY, Lino W. The Healthy Eating Index: 1999 - 2000. In: U.S. Department of Agriculture CfNPaP, editor. 2002

33. Hacker AN, Fung EB, King JC. Role of calcium during pregnancy: maternal and fetal needs. Nutr Rev. 2012:70(7):397-409.

34. Jiang X, West AA, Caudill MA. Maternal choline supplementation: a nutritional approach for improving offspring health? Trends Endocrinol Metab. 2014;25(5):263-73. 
35. Qiu C, Coughlin KB, Frederick IO, Sorensen TK, Williams MA. Dietary fiber intake in early pregnancy and risk of subsequent preeclampsia. Am J Hypertens. 2008;21(8):903-9.

36. Reynolds CM, Vickers MH, Harrison CJ, Segovia SA, Gray C. High fat and/or high salt intake during pregnancy alters maternal meta-inflammation and offspring growth and metabolic profiles. Physiol Rep. 2014;1:2(8). Pubmed Central PMCID: 4246600

37. IOM. Dietary Reference Intakes Tables and Applications 2013 November 16, 2015. Available from: https://fnic.nal.usda.gov/dietary-guidance/ dietary-reference-intakes/dri-tables-and-application-reports. Accessed 16 July 2016.

38. Poston L, Bell R, Croker H, Flynn AC, Godfrey KM, Goff L, et al. Effect of a behavioural intervention in obese pregnant women (the UPBEAT study): a multicentre, randomised controlled trial. Lancet Diab Endocrinol. 2015;3(10): 767-77.

39. Skouteris H, McPhie S, Hill B, McCabe M, Milgrom J, Kent B, et al. Health coaching to prevent excessive gestational weight gain: A randomizedcontrolled trial. Br J Health Psychol. 2016;21 (1):31-51.

40. Lally P, Chipperfield A, Wardle J. Healthy habits: efficacy of simple advice on weight control based on a habit-formation model. Int J Obes (Lond). 2008;32(4):700-7.

41. Merriam PA, Ma Y, Olendzki BC, Schneider KL, Li W, Ockene IS, et al. Design and methods for testing a simple dietary message to improve weight loss and dietary quality. BMC Med Res Methodol. 2009;9:87. Pubmed Central PMCID: 2811706

42. Olander EK, Atkinson L, Edmunds JK, French DP. The views of pre- and post-natal women and health professionals regarding gestational weight gain: An exploratory study. Sex Reprod Healthcare. 2011;2(1):43-8

43. Whitaker KM, Wilcox S, Liu J, Blair SN, Pate RR. Pregnant women's perceptions of weight gain, physical activity, and nutrition using Theory of Planned Behavior constructs. J Behav Med. 2016;39(1):41-54. Pubmed Central PMCID: 4753045

44. Darmon N, Drewnowski A. Does social class predict diet quality? Am J Clin Nutr. 2008:87(5):1107-17.

45. Lin $\mathrm{H}$, Carr KA, Fletcher KD, Epstein LH. Food reinforcement partially mediates the effect of socioeconomic status on body mass index. Obesity. 2013;21(7):1307-12. Pubmed Central PMCID: 3742692.

46. Baker EA, Schootman M, Barnidge E, Kelly C. The role of race and poverty in access to foods that enable individuals to adhere to dietary guidelines. Prev Chronic Dis. 2006;3(3):A76. Pubmed Central PMCID: 1636719.

47. Diez-Roux AV, Nieto FJ, Caulfield L, Tyroler HA, Watson RL, Szklo M. Neighbourhood differences in diet: the Atherosclerosis Risk in Communities (ARIC) Study. J Epidemiol Community Health. 1999;53(1):55-63. Pubmed Central PMCID: 1756776.

48. Morland K, Wing S, Diez RA. The contextual effect of the local food environment on residents' diets: the atherosclerosis risk in communities study. Am J Public Health. 2002;92(11):1761-7. Pubmed Central PMCID: 1447325.

\section{Submit your next manuscript to BioMed Central and we will help you at every step:}

- We accept pre-submission inquiries

- Our selector tool helps you to find the most relevant journal

- We provide round the clock customer support

- Convenient online submission

- Thorough peer review

- Inclusion in PubMed and all major indexing services

- Maximum visibility for your research

Submit your manuscript at www.biomedcentral.com/submit

) Biomed Central 